\title{
Weekday of Surgery Affects Postoperative Complications and Long-Term Survival of Chinese Gastric Cancer Patients after Curative Gastrectomy
}

\author{
Rong Li, ${ }^{1,2}$ Ai-min Leng, ${ }^{3}$ Ting Liu, ${ }^{3}$ Yan-wu Zhou, ${ }^{4}$ Jun-xian Zeng, ${ }^{5}$ Xiao-ming Liu, \\ Ting-zi Hu, ${ }^{1,2}$ Xiao-xia Jiang, ${ }^{1,2}$ Lin-fang Zhang, ${ }^{1,2}$ and Can-Xia Xu ${ }^{1,2}$ \\ ${ }^{1}$ Department of Gastroenterology, Third Xiangya Hospital of Central South University, Changsha, Hunan 410013, China \\ ${ }^{2}$ Hunan Key Laboratory of Nonresolving Inflammation and Cancer, Changsha, Hunan 410013, China \\ ${ }^{3}$ Department of Gastroenterology, Xiangya Hospital of Central South University, Changsha, Hunan 410008, China \\ ${ }^{4}$ Department of Thoracic Surgery, Xiangya Hospital of Central South University, Changsha, Hunan 410008, China \\ ${ }^{5}$ Department of General Surgery, Third Xiangya Hospital of Central South University, Changsha, Hunan 410013, China
}

Correspondence should be addressed to Can-xia Xu; xucanxia2000@163.com

Received 19 November 2016; Accepted 2 April 2017; Published 18 April 2017

Academic Editor: Christian Schwentner

Copyright (c) 2017 Rong Li et al. This is an open access article distributed under the Creative Commons Attribution License, which permits unrestricted use, distribution, and reproduction in any medium, provided the original work is properly cited.

\begin{abstract}
Many factors have been reported to affect the long-term survival of gastric carcinoma patients after gastrectomy; the present study took the first attempt to find out the potential role of weekday carried out surgery in the postoperative prognosis of gastric cancer patients. 463 gastric cancer patients have been followed up successfully. Pearson $\chi^{2}$ test was used for univariate analyses. Survival curves were constructed by using Kaplan-Meier method and evaluated by using the log-rank test. The Cox proportional hazard regression model was used to find out the risk factors, and subgroup analysis was conducted to rule out confounding factors. We found that the patients who underwent gastrectomy on the later weekday (Wednesday-Friday) more easily suffered from a higher postoperative morbidity. Weekday of surgery was one of the independent indicators for the prognosis of patients after gastric cancer surgery. However, the role of weekday of surgery was significantly weakened in the complications group. In conclusion, surgery performed in the later weekday was more likely to lead to increased postoperative complications and an unfavorable role in prognosis of Chinese gastric cancer patients after curative gastrectomy.
\end{abstract}

\section{Introduction}

With a mortality of 498.0/100,000, gastric cancer has been regarded as the forth leading cancer-related lethal disease and ranks as one of the 5 most commonly diagnosed cancers in China [1]. Though early diagnosis of gastric carcinoma has gained great progress because of the rapid advancement in the endoscopic technique, the outcome of stomach malignancy continues to be unsatisfactory [1]. Consequently, investigators are engaged in finding out which controllable factors really affect the prognosis, thus to improve the current dismal situation of gastric cancer. Long-term outcome after gastrectomy relies on several universal well-known factors such as tumor differentiation, numbers of involved lymph nodes,
TNM stage, operation technique, and adjuvant therapy $[2,3]$. Even the extreme body mass index and hypoproteinemia are reported to indicate a poorer prognosis [4-6]. Currently, gastrectomy is still the basic and widely accepted treatment for curable gastric carcinoma. Though surgery always produces more satisfactory outcomes in experienced hands and specialized centers [7], its accompanying postoperative complications are reported to increase the postoperative quality and survival differently [8-10]. It indicates that control of postoperative complications would significantly improve the disappointing survival.

Interestingly, previous researches have also reported that the weekday choice of surgery did play a significant role in the prognosis after tumorectomy. In an analysis of 4, 133, 346 
inpatient admissions for elective operating room procedures, Aylin et al. reported that surgery in the later week or weekend increased postoperative 30-day mortality [11]. The 5-year mortality of curable esophageal cancer was increased after surgery later in the week $[12,13]$. However, whether weekday of surgery plays a role in postoperative complications or prognosis of gastric cancer is not clear.

Therefore, the present study was aimed at identifying the potential role of weekday of surgery in postoperative complications as well as the long-term survival after gastrectomy. In this study, we found that patients who underwent surgery on the later weekday (Wednesday to Friday) more easily suffered from an increased postoperative morbidity and a poorer prognosis in gastric cancer than those in the earlier weekday. Taken together, our results indicated that more strict postoperative complications control and proper scheduling would improve the prognosis of gastric cancer.

\section{Materials and Methods}

2.1. Patient Selection Criterion. Firstly, we have gained the approval of the Institutional Review Board of Third Xiangya Hospital of Central South University, Hunan, China. From January 1, 2012, we retrospectively collected the medical and clinicopathological information of the patients who were diagnosed with gastric carcinoma and underwent gastrectomy during the period from January 1, 2004, to January 1, 2010, at the Department of Gastrointestinal Surgery, Xiangya Third Hospital. The details for recruited patients have referred to the previously described methods [14]. The selection criteria were as follows: (1) patient was diagnosed with gastric carcinoma histologically confirmed by at least 2 pathologists, without distant metastasis and received curative gastrectomy; (2) lymphadenectomy was performed with R0 margin if necessary; (3) the details regarding clinicopathological features, postoperative complications, and follow-up information were available and intact; (4) life span after operation was more than 60 days. As a consequence, there were 463 patients enrolled in our analysis. The clinicopathological data were collected in detail from the original medical records as summarized in the Table 1; they were grouped by clinical, pathological, and therapy-related characters. Additionally, the tumor-node-metastases (TNM) stage was classified according to American Joint Committee on Cancer, AJCC 7th edition [15]. All the personal information was concealed to ensure the privacy. The design and methods of this study were in accordance with the requirements of related regulations and procedures (such as GCP, ICH-GCP) as well as the ethical principles. The IRB of Third Xiangya Hospital, Central South University, has approved the research to be conducted and the approval number was 2016-S232. All authors had access to information that could identify individual participants during or after data collection.

2.2. Complications. Any deviation from the normal course within 30 days after operation was seen as a postoperative complication, which was recorded in detail (medical, surgical, infectious complications, etc.). According to the revised Clavien-Dindo classification system [16], the concreteness was as follows: Grade I complications include any deviation from the uneventful postoperative course without the need for pharmacological treatment or surgical, endoscopic, and radiological interventions, except the drugs as follows: antipyretics, antiemetics, analgesics, electrolytes, diuretics, and physiotherapy. Wound infections and nutritional support were also included. Grade II complications could be treated solely by drugs, blood transfusion, and parenteral nutrition. Grade III complication required endoscopic, radiological, or surgical intervention. Grade IV included lifethreatening complications (including CNS complications) which required intensive-care unit management. Grade III or Grade IV altogether was regarded as severe complications. Grade V meant death of the patient, which was excluded from our analysis. The details were displayed in Table 2.

2.3. Follow-Up. The objects enrolled were followed up every 3 months during the first postoperative year and at least 6 months afterward for survival and recurrence inquiry. The data were obtained by telephone, letter, message, and return visitation. The follow-up data were calculated from the date of surgery to the death or the end of data collection. The followup was stopped on January 1, 2015.

2.4. Statistics. The SPSS software (version 16.0, Chicago, IL) and GraphPad prism 5.0 were used to complete all statistical analyses and graphics. To clarify the potential variables affecting the postoperative morbidity, the clinical, pathological, and therapy-related characters were grouped and compared between those who underwent complications or not. A Pearson $\chi^{2}$ test or Fisher's exact test was used for univariate analyses where appropriate. Survival curves were constructed by using the Kaplan-Meier method and evaluated by using the log-rank test. Subgroup analysis was conducted to rule out confounding factors. The Cox proportional hazard regression model was used to identify factors that were independently associated with overall survival and disease-free survival. The variables with a $p$ value less than 0.05 in the univariate analysis were considered potential candidates for the main effects and were evaluated in a stepwise multivariate Cox analysis. In multivariate analysis, $p$ values less than 0.05 were considered statistically significant.

\section{Results}

(1) Patients Who Received Gastrectomy in the Later Weekday Were Prone to Suffering from the Postoperative Complications. From January, 2004, to January, 2010, a total of 495 consecutive gastric carcinoma patients who underwent gastrectomy were enrolled; among them 32 patients died in 30 days (Grade $\mathrm{V},<30$ days) after operation, and they have been excluded in this analysis. As shown in Table 1, there were 198 patients took surgery on the earlier weekday (Monday or Tuesday) and 265 patients on the later weekday (Wednesday-Friday). The patients who underwent gastrectomy on the later weekday more easily suffered from a longer operative duration or more operative related blood loss. Moreover, the patients who took gastrectomy on the later weekday had a higher risk of suffering from postoperative complications. However, 
TABLE 1: The clinicopathological characters of 463 patients subjected to curative gastrectomy.

\begin{tabular}{|c|c|c|c|c|}
\hline Characteristics & $n$ & Monday-Tuesday & Wednesday-Friday & $p$ value \\
\hline \multicolumn{5}{|l|}{ Gender } \\
\hline Male & 224 & 102 & 122 & \multirow{2}{*}{0.26} \\
\hline Female & 239 & 96 & 143 & \\
\hline \multicolumn{5}{|l|}{ Age (year) } \\
\hline$\leq 65$ & 265 & 123 & 142 & \multirow{2}{*}{0.066} \\
\hline$>65$ & 198 & 75 & 123 & \\
\hline \multicolumn{5}{|l|}{ Smoking history } \\
\hline No & 196 & 80 & 116 & \multirow{2}{*}{0.506} \\
\hline Yes & 267 & 118 & 149 & \\
\hline \multicolumn{5}{|l|}{ BMI } \\
\hline Low BMI $\left(<18 \mathrm{~kg} / \mathrm{m}^{2}\right)$ & 175 & 73 & 102 & \multirow{3}{*}{0.936} \\
\hline Normal BMI $\left(18-25 \mathrm{~kg} / \mathrm{m}^{2}\right)$ & 247 & 107 & 140 & \\
\hline High BMI $\left(>25 \mathrm{~kg} / \mathrm{m}^{2}\right)$ & 41 & 18 & 23 & \\
\hline \multicolumn{5}{|l|}{ Comorbidities } \\
\hline No & 340 & 139 & 191 & \multirow{2}{*}{0.670} \\
\hline Yes & 123 & 49 & 74 & \\
\hline \multicolumn{5}{|l|}{ ASA $(0.000)$} \\
\hline I & 340 & 139 & 191 & \multirow{3}{*}{0.850} \\
\hline II & 101 & 41 & 60 & \\
\hline III & 22 & 8 & 14 & \\
\hline \multicolumn{5}{|l|}{ Differentiation } \\
\hline Undifferentiated & 23 & 10 & 13 & \multirow{2}{*}{0.611} \\
\hline Differentiated & 440 & 188 & 252 & \\
\hline \multicolumn{5}{|l|}{ Tumor size } \\
\hline$\leq 5 \mathrm{~cm}$ & 322 & 136 & 186 & \multirow{2}{*}{0.76} \\
\hline$>5 \mathrm{~cm}$ & 141 & 62 & 79 & \\
\hline \multicolumn{5}{|l|}{ Hypoproteinemia } \\
\hline No & 250 & 115 & 135 & \multirow{2}{*}{0.133} \\
\hline Yes & 213 & 83 & 130 & \\
\hline \multicolumn{5}{|l|}{ Increased CEA } \\
\hline No & 263 & 108 & 155 & \multirow{2}{*}{0.448} \\
\hline Yes & 200 & 90 & 110 & \\
\hline \multicolumn{5}{|l|}{ T classification } \\
\hline $\mathrm{T} 1$ & 13 & 5 & 8 & \multirow{4}{*}{0.345} \\
\hline $\mathrm{T} 2$ & 135 & 57 & 78 & \\
\hline $\mathrm{T} 3$ & 276 & 114 & 162 & \\
\hline $\mathrm{T} 4$ & 39 & 22 & 17 & \\
\hline $\mathrm{N}$ classification & & & & \\
\hline No & 198 & 96 & 102 & \\
\hline N1 & 195 & 77 & 118 & 0158 \\
\hline $\mathrm{N} 2$ & 53 & 18 & 35 & \\
\hline N3 & 17 & 7 & 10 & \\
\hline TNM stage & & & & \\
\hline I & 73 & 34 & 39 & \\
\hline II & 321 & 132 & 189 & 0.561 \\
\hline III & 69 & 32 & 37 & \\
\hline Procedure (whether laparoscop & & & & \\
\hline No & 397 & 167 & 230 & 0.502 \\
\hline Yes & 66 & 31 & 35 & 0.502 \\
\hline
\end{tabular}


TABLE 1: Continued.

\begin{tabular}{|c|c|c|c|c|}
\hline Characteristics & $n$ & Monday-Tuesday & Wednesday-Friday & $p$ value \\
\hline \multicolumn{5}{|l|}{ Medical team } \\
\hline Group 1 & 167 & 69 & 98 & \multirow{3}{*}{0.757} \\
\hline Group 2 & 155 & 70 & 85 & \\
\hline Group 3 & 141 & 59 & 82 & \\
\hline \multicolumn{5}{|l|}{ Chemotherapy } \\
\hline No & 106 & 49 & 57 & \multirow{2}{*}{0.412} \\
\hline Yes & 357 & 149 & 208 & \\
\hline \multicolumn{5}{|l|}{ Operation time } \\
\hline$\leq 240 \min$ & 354 & 164 & 190 & \multirow{2}{*}{0.006} \\
\hline$>240 \mathrm{~min}$ & 109 & 34 & 75 & \\
\hline \multicolumn{5}{|c|}{ Intraoperative blood loss } \\
\hline$\leq 500 \mathrm{ml}$ & 391 & 176 & 215 & \multirow{2}{*}{0.027} \\
\hline$>500 \mathrm{ml}$ & 72 & 22 & 50 & \\
\hline \multicolumn{5}{|c|}{ Postoperative complications } \\
\hline Without & 362 & 164 & 198 & \multirow{2}{*}{0.041} \\
\hline With & 101 & 34 & 67 & \\
\hline \multicolumn{5}{|c|}{ Postoperative complications } \\
\hline Grade 0 & 362 & 164 & 198 & \multirow{5}{*}{0.072} \\
\hline Grade I & 45 & 19 & 26 & \\
\hline Grade II & 28 & 9 & 19 & \\
\hline Grade III & 17 & 5 & 12 & \\
\hline Grade IV & 11 & 1 & 10 & \\
\hline \multicolumn{5}{|l|}{ Postoperative CRP } \\
\hline Normal CRP & 285 & 121 & 164 & \multirow{2}{*}{0.923} \\
\hline Increased CRP & 178 & 77 & 101 & \\
\hline
\end{tabular}

TABLE 2: Patients who took surgery on the later weekday were more inclined to get severe complications (Grade III + Grade IV).

\begin{tabular}{lccccc}
\hline & Grade 0 & Grade I & Grade II & Grade III & Grade IV \\
\hline Monday & 107 & 11 & 4 & 5 & 0 \\
Tuesday & 57 & 8 & 5 & 0 & 1 \\
Wednesday & 60 & 4 & 4 & 5 & 0 \\
Thursday & 60 & 7 & 5 & 3 & 6 \\
Friday & 78 & 15 & 10 & 4 & 4 \\
\hline
\end{tabular}

we found that there was no major difference in distribution of general state (age, gender, smoking history, and BMI), disease-state (comorbidities and ASA grade), tumor state (differentiation, size, serum CEA level, and TNM stage), and therapeutic schedule (operation procedure and adjuvant therapy) between the two groups.

There were about $21.8 \%(101 / 463)$ patients who experienced postoperative complications eventually. 34 patients in the earlier weekday had postoperative complications and 67 in the later weekday. They were classified into 4 groups according to the revised Clavien-Dindo classification system [16] (Grade I, $n=45$; Grade II, $n=28$; Grade III, $n=17$; Grade IV, $n=11$ ). Furthermore, we found that the postoperative morbidity was increased gradually from Monday to Friday. Though not statistically significant, the degree of complications severity increased from Wednesday through Thursday to Friday. Moreover, the severe complications (Grade III + Grade IV) mainly focused on Thursday or Friday (Table 2).

(2) Weekday of Surgery Was Associated with the LongTerm Survival in Gastric Cancer Patients after Gastrectomy. To assess the potential influential factors in gastric cancer prognosis, Cox proportional hazards regression model was introduced. Univariate analyses (Table 3) displayed that the postoperative 5-year survival was associated with comorbidities, tumor size, operative time, intraoperative blood loss, postoperative serum CRP, TNM stage, adjuvant therapy, and postoperative complications. It is worth mentioning that surgery in the later weekday also promoted an unfavorable prognosis after gastrectomy. Then, the variables with a $p$ value less than 0.05 in the univariate analysis were further pooled in a multivariate analysis. On multivariate survival analysis, weekday of surgery $(p<0.001)$, postoperative complications $(p<0.001)$, postoperative serum CRP $(p=$ $0.026)$, and TNM stage $(p=0.033)$ reached significance for overall survival time (OS) (Table 4). Surgery performed in the later weekday decreased the 5-year overall survival rate 1.721 times when compared to the surgery in the earlier weekday. For analysis of disease-free survival time (DFS), weekday of surgery $(p<0.001)$, postoperative complications $(p<0.001)$, postoperative serum CRP $(p=0.004)$, and TNM stage $(p=0.027)$ also reached significance in 
TABLE 3: Univariate cox analysis of overall and disease-free survival in 463 patients after gastrectomy.

\begin{tabular}{|c|c|c|c|c|}
\hline \multirow{2}{*}{ Characteristics } & \multicolumn{2}{|c|}{ Overall survival } & \multicolumn{2}{|c|}{ Disease-free survival } \\
\hline & $\operatorname{HR}(95 \% \mathrm{CI})$ & $p$ Value & $\operatorname{HR}(95 \% \mathrm{CI})$ & $p$ Value \\
\hline Gender (female/male) & $1.190(0.956-1.483)$ & 0.120 & $1.158(0.939-1.428)$ & 0.171 \\
\hline Age (year) $(>65 / \leq 65)$ & $1.126(0.903-1.405)$ & 0.293 & $1.153(0.933-1.424)$ & 0.187 \\
\hline BMI (abnormal BMI/normal BMI) & $1.093(0.878-1.361)$ & 0.426 & $1.079(0.875-1.331)$ & 0.477 \\
\hline Smoking history (yes/no) & $1.008(0.808-1.258)$ & 0.945 & $1.015(0.821-1.254)$ & 0.893 \\
\hline Comorbidities (with/without) & $1.196(0.936-1.527)$ & 0.152 & $1.282(1.018-1.616)$ & 0.035 \\
\hline ASA (III/I + II) & $1.277(0.784-2.082)$ & 0.326 & $1.564(0.996-2.457)$ & 0.052 \\
\hline Tumor Size $(\geq 5 \mathrm{~cm} /<5 \mathrm{~cm})$ & $1.180(0.932-1.494)$ & 0.169 & $1.320(1.057-1.649)$ & 0.015 \\
\hline Tumor differentiation (yes/no) & $0.818(0.502-1.333)$ & 0.420 & $0.856(0.526-1.394)$ & 0.533 \\
\hline Increased serum CEA (yes/no) & $0.914(0.732-1.140)$ & 0.425 & $0.960(0.777-1.186)$ & 0.704 \\
\hline Hypoalbuminemia (yes/no) & $1.032(0.828-1.286)$ & 0.779 & $0.971(0.787-1.198)$ & 0.783 \\
\hline Laparoscopic-assisted (yes/no) & $0.883(0.640-1.218)$ & 0.448 & $0.952(0.705-1.284)$ & 0.745 \\
\hline Operation time $(\geq 240 \mathrm{~min} /<240 \mathrm{~min})$ & $1.653(1.295-2.112)$ & $<0.001$ & $1.495(1.178-1.896)$ & 0.001 \\
\hline Operative blood loss $(\geq 500 \mathrm{ml} /<500 \mathrm{ml})$ & $1.604(1.212-2.125)$ & 0.001 & $1.509(1.149-1.983)$ & 0.003 \\
\hline T classification & & $<0.001$ & & $<0.001$ \\
\hline$(\mathrm{T} 2 / \mathrm{T} 1)$ & $1.723(0.697-4.260)$ & 0.239 & $1.239(0.574-2.678)$ & 0.585 \\
\hline (T3/T1) & $2.869(1.181-6.967)$ & 0.020 & $2.042(0.962-4.334)$ & 0.063 \\
\hline$(\mathrm{T} 4 / \mathrm{T} 1)$ & $4.834(1.889-12.366)$ & 0.001 & $3.105(1.378-6.993)$ & 0.006 \\
\hline $\mathrm{N}$ classification & & $<0.001$ & & $<0.001$ \\
\hline$(\mathrm{N} 1 / \mathrm{N} 0)$ & $2.005(1.571-2.558)$ & $<0.001$ & $2.280(1.801-2.886)$ & $<0.001$ \\
\hline$(\mathrm{N} 2 / \mathrm{N} 0)$ & $2.184(1.521-3.136)$ & $<0.001$ & $2.805(1.998-3.937)$ & $<0.001$ \\
\hline (N3/N0) & $3.526(1.984-6.268)$ & $<0.001$ & $3.900(2.239-6.792)$ & $<0.001$ \\
\hline TNM stage & & $<0.001$ & & $<0.001$ \\
\hline$(\mathrm{II} / \mathrm{I})$ & $2.796(1.877-4.167)$ & $<0.001$ & $2.929(2.005-4.279)$ & $<0.001$ \\
\hline$(\mathrm{III} / \mathrm{I})$ & $7.397(4.679-11.692)$ & $<0.001$ & $7.874(5.083-12.198)$ & $<0.001$ \\
\hline Chemotherapy (no/yes) & $1.578(1.231-2.023)$ & $<0.001$ & $1.771(1.396-2.247)$ & $<0.001$ \\
\hline Postoperative complications (yes/no) & $3.617(2.825-4.631)$ & $<0.001$ & $3.395(2.666-4.324)$ & $<0.001$ \\
\hline Weekday (late/early) & $1.574(1.255-1.974)$ & $<0.001$ & $1.488(1.201-1.844)$ & $<0.001$ \\
\hline Increased postoperative CRP (yes/no) & $1.857(1.490-2.315)$ & $<0.001$ & $1.856(1.503-2.293)$ & $<0.001$ \\
\hline
\end{tabular}

the multivariate survival analysis Cox proportional hazards regression model. Similarly, the surgery performed in the later weekday increased 5-year relapse rate by 1.693 times (Table 4). It indicated that surgery in the later weekday promoted a worse outcome in gastric cancer patients after gastrectomy.

(3) Patients Who Received Gastrectomy in the Later Weekday or Suffered from Postoperative Complications Had a Shorter OS and DFS. Moreover, the Kaplan-Meier analysis showed that patients who undertook gastrectomy in the later weekday had a shorter survival time compared to those in Monday or Tuesday (OS: 44 versus 50 months, $p=0.015$; DFS: 36 versus 44 months, $p=0.011$; Figures $1(\mathrm{a})$ and $1(\mathrm{~b})$ ). Moreover, compared with the surgery carried out on Monday, the surgery on Friday increased relapse and death rate (HR (hazard ratio $)=1.415,95 \% \mathrm{CI}, 1.054-1.901$ and $\mathrm{HR}=1.559$, 95\% CI, 1.142-2.128), respectively (Table 5). As we all know, it needs more time and energy to complete a surgery for a tumor in advanced TNM stage; we also demonstrated that risk estimates of gastric cancer surgery in the later weekday were evident for tumor in stage I or II, but not for stage III
(Supplementary Figure 1 in Supplementary Material available online at https://doi.org/10.1155/2017/5090534).

As shown in the multivariate analysis, the postoperative complication was also an independent indicator for the gastric cancer outcome. Furthermore, gastric cancer patients with postoperative complications had much shorter OS than those without complications (median survival time, 34 versus 49 months, $p<0.001$; Figure $1(\mathrm{c})$ ) and displayed an increased mortality (adjusted HR $=3.169$, 95\% CI, 2.388-4.204, Table 4). Similarly, gastric cancer patients with postoperative complications had a shorter DFS (median survival time, 28 versus 43 months, $p<0.001$; Figure $1(\mathrm{~d})$ ) than those without complications and showed an increased postoperative relapse rate (adjusted $\mathrm{HR}=2.826,95 \% \mathrm{CI}, 2.134-3.740$, Table 4). Moreover, when postoperative complication was categorized into the 5 degrees, the HR for postoperative relapse rate or mortality gradually increased from Grade 0 to Grade IV (Supplementary Table 1).

Similarly, in order to clarify the potential role of postoperative complication in different TNM stage, we conducted a subgroup analysis, and we found a significant role of 
TABLE 4: Multivariate Cox regression analysis of overall and disease-free survival in 463 patients after gastrectomy.

\begin{tabular}{|c|c|c|c|c|}
\hline \multirow{2}{*}{ Characteristics } & \multicolumn{2}{|c|}{ Overall survival } & \multicolumn{2}{|c|}{ Disease-free survival } \\
\hline & HR $(95 \%$ CI $)$ & $p$ value & $\operatorname{HR}(95 \% \mathrm{CI})$ & $p$ value \\
\hline Comorbidities (with/without) & - & - & $0.886(0.636-1.233)$ & 0.472 \\
\hline Tumor size $(\geq 5 \mathrm{~cm} /<5 \mathrm{~cm})$ & - & - & $1.055(0.766-1.453)$ & 0.741 \\
\hline Operation time $(\geq 240 \mathrm{~min} /<240 \mathrm{~min})$ & $0.869(0.582-1.299)$ & 0.494 & $0.770(0.520-1.139)$ & 0.191 \\
\hline Operative blood loss $(\geq 500 \mathrm{ml} /<500 \mathrm{ml})$ & $1.023(0.691-1.514)$ & 0.909 & $0.968(0.662-1.417)$ & 0.869 \\
\hline T classification & & 0.017 & & 0.018 \\
\hline$(\mathrm{T} 2 / \mathrm{T} 1)$ & $2.315(0.902-5.945)$ & 0.081 & $1.517(0.670-3.433)$ & 0.318 \\
\hline (T3/T1) & $3.691(1.373-9.921)$ & 0.010 & $2.528(1.062-6.020)$ & 0.036 \\
\hline$(\mathrm{T} 4 / \mathrm{T} 1)$ & $4.001(1.231-13.007)$ & 0.021 & $2.677(0.920-7.789)$ & 0.071 \\
\hline $\mathrm{N}$ classification & & $<0.001$ & & $<0.001$ \\
\hline$(\mathrm{N} 1 / \mathrm{N} 0)$ & $1.790(1.350-2.374)$ & $<0.001$ & $1.988(1.515-2.608)$ & $<0.001$ \\
\hline (N2/N0) & $1.953(1.144-3.333)$ & 0.014 & $2.510(1.515-4.157)$ & $<0.001$ \\
\hline (N3/N0) & $2.838(1.325-6.078)$ & 0.007 & $2.954(1.422-6.138)$ & 0.004 \\
\hline TNM stage & & 0.016 & & 0.005 \\
\hline$(\mathrm{II} / \mathrm{I})$ & $1.182(0.674-2.075)$ & 0.560 & $1.118(0.654-1.912)$ & 0.684 \\
\hline$(\mathrm{III} / \mathrm{I})$ & $2.520(1.078-5.894)$ & 0.033 & $2.536(1.114-5.773)$ & 0.027 \\
\hline Postoperative complications (with/without) & $3.169(2.388-4.204)$ & $<0.001$ & $2.826(2.134-3.740)$ & $<0.001$ \\
\hline Weekday (late/early) & $1.721(1.343-2.205)$ & $<0.001$ & $1.693(1.331-2.153)$ & $<0.001$ \\
\hline Chemotherapy (no/yes) & $1.087(0.830-1.423)$ & 0.546 & $1.204(0.928-1.562)$ & 0.161 \\
\hline Increased postoperative CRP (yes/no) & $1.399(1.042-1.879)$ & 0.026 & $1.533(1.150-2.045)$ & 0.004 \\
\hline
\end{tabular}

TABLE 5: The death and relapse rate after gastric cancer were increased from Monday to Friday through Wednesday.

\begin{tabular}{|c|c|c|c|c|}
\hline $\begin{array}{l}\text { Weekday of } \\
\text { surgery }\end{array}$ & $p(\mathrm{OS})$ & HR (95\% CI) & $p(\mathrm{DFS})$ & HR (95\% CI) \\
\hline Monday & 0.002 & 1 & 0.003 & 1 \\
\hline Tuesday & 0.974 & $\begin{array}{c}1.006 \\
(0.695-1.457)\end{array}$ & 0.823 & $\begin{array}{c}0.961 \\
(0.677-1.364)\end{array}$ \\
\hline Wednesday & 0.036 & $\begin{array}{c}1.455 \\
(1.025-2.065)\end{array}$ & 0.121 & $\begin{array}{c}1.302 \\
(0.933-1.819)\end{array}$ \\
\hline Thursday & 0.001 & $\begin{array}{c}1.724 \\
(1.238-2.400)\end{array}$ & 0.001 & $\begin{array}{c}1.722 \\
(1.259-2.355)\end{array}$ \\
\hline Friday & 0.005 & $\begin{array}{c}1.559 \\
(1.142-2.128)\end{array}$ & 0.021 & $\begin{array}{c}1.415 \\
(1.054-1.901)\end{array}$ \\
\hline
\end{tabular}

postoperative complications in predicting prognosis in stages I and II, but not in stage III (Supplementary Figure 2).

(4) Surgery Performed in the Later Weekday Might Deteriorate the Long-Term Survival by Promoting the Postoperative Complications. As noted above, patients who got gastrectomy in the later weekday showed higher risk of suffering from postoperative complication and a poor prognosis (Table 1, Figure 1). To clarify the inner relationship between weekday of surgery and postoperative complications in prognosis, a subgroup analysis was conducted. When complication condition was confined, the effect of weekday of surgery was limited in patients with complication (Figure 2, Supplementary Table 2). However, when we confined the weekday of surgery, we found that the occurrence of postoperative complications promoted a much shorter survival time regardless of the weekday of surgery (Figure 3, Supplementary Table 3). It indicated that the postoperative complication was a valid potential prognostic indicator; it might conceal the effect of the weekday of surgery on prognosis after surgery for gastric cancer to some extent.

\section{Discussion}

Due to the current unsatisfactory outcome of gastric cancer patients, clinical doctors and researchers have put a lot of efforts to improve survival in resectable gastric cancer [17, 18]. Postoperative complications have been reported to not only prolong the postoperation hospitalization duration and increased costs [19], but also promote unfavorable prognosis $[8-10,20]$. Surgical complication is tightly related to the quality of the operative technique and the surgeon's caseload; the morbidity is higher in inexperienced hands [7, 21]. As multidisciplinary collaboration could be performed in a general hospital, the occurrence of surgical complications was relatively low in our institution, compared to morbidity from $15.5 \%$ to $24.7 \%$ [6]. However, in the present study, postoperative complication was also demonstrated to be an independent indicator of poor prognosis after gastric cancer surgery. As discussed previously, postoperative complications commonly promoted a persistent period of immunosuppression and allowed residual tumor cells to survive in the host, which lead to an earlier cancer recurrence [22, 23]. Moreover, excessively activated inflammatory response was also an important aspect. Like in our results, postoperative complications always accompanied with an increased serum CRP, the infectious complications potentiated proinflammatory cytokine cascades, including interleukins 1, 8 and tumor 


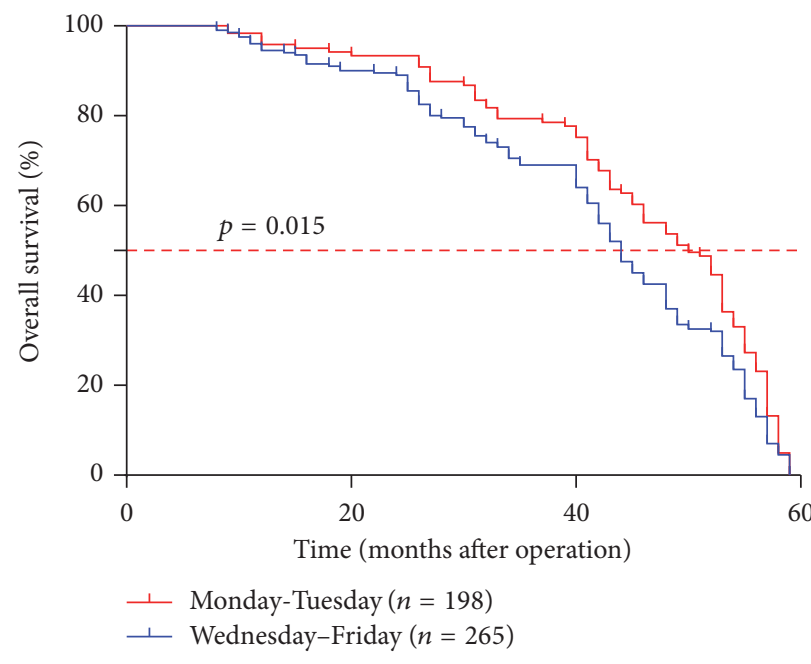

(a)

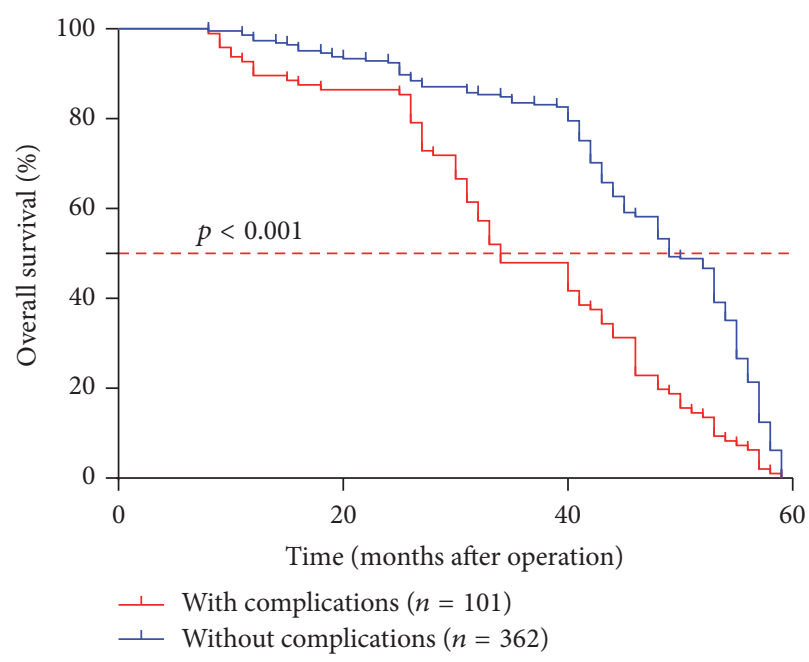

(c)

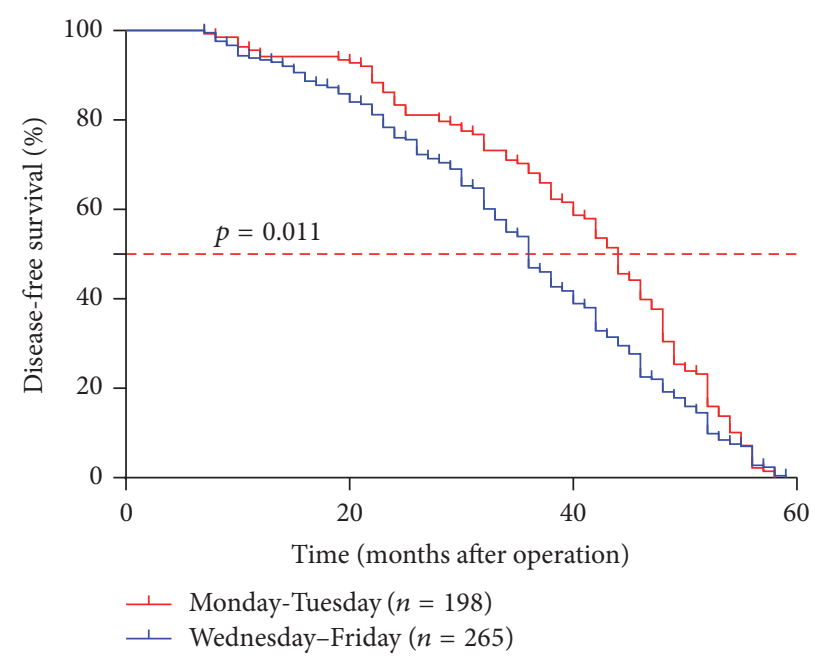

(b)

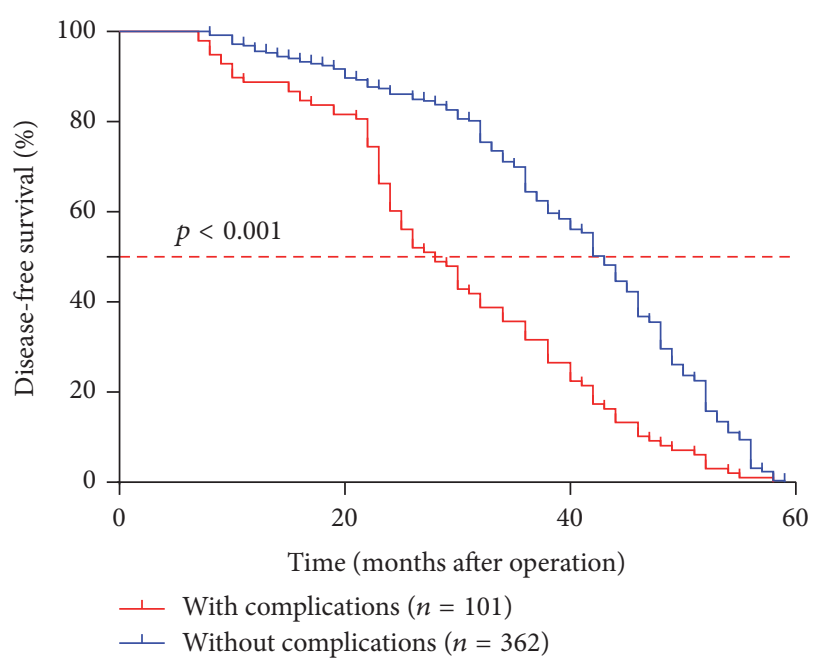

(d)

FIGURE 1: Weekday of surgery or postoperative complications influenced the overall survival time and disease-free survival time after gastrectomy. The 5-year overall survival rate and disease-free survival rate was much lower in the patients who underwent surgery on Wednesday to Friday than those on Monday to Tuesday (a, b). Similarly, the patients with postoperative complications shared a much shorter overall survival and disease-free survival time than those without complications (c, d).

necrosis factor-alpha. These activated cytokines could disable the function and regulation of cytotoxic T-lymphocytes, natural killer cells, and antigen-presenting cells [24-26].

Therefore, identifying factors related to complications would also aid in the successful treatment of gastrectomy patients. Interestingly, we found that the weekday of surgery was associated with the occurrence of postoperative complications. Previously, the "weekend effect" has been proposed that postoperative complications might be less well handled during weekends [27]. Some research has also revealed that surgery in earlier weekday was regarded as beneficial in short term (30 days) after elective surgery [11, 13]. Esophageal cancer surgery was suggested to be carried out earlier in the week, for the increased 5-year mortality of potentially curable esophageal cancer after surgery later in the week [12]. Moreover, our present study has demonstrated that surgery performed in the later weekday was an indicator of the adverse outcome after gastrectomy in gastric cancer patients. To the best of our knowledge, this is the first report to address the potential influence of weekday of gastrectomy in relation to postoperative complications and long-term survival in gastric cancer. Moreover, surgery performed in the later weekday deteriorated the long-term survival by promoting the postoperative complications.

Factors like the age or tumor stage did not influence the choice of operation day in this study; however, the occurrence of postoperative complications and complications severity were affected by the weekday of surgery. All these might be interpreted by the hypothesis that the health care services were of lower quality during the later weekday. Firstly, the surgical precision might be deteriorated to some extent due to the workload of surgeons and surgical team; secondly, the 


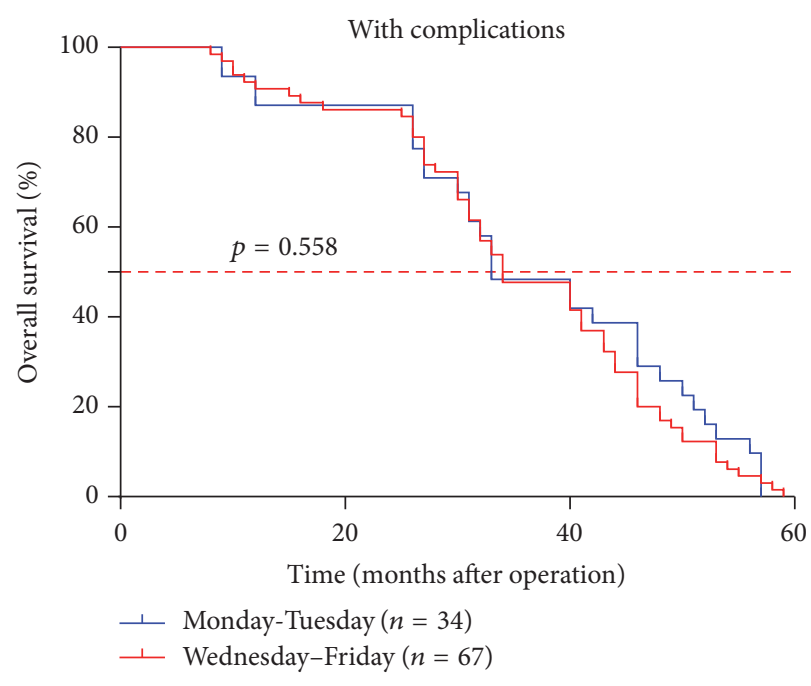

(a)

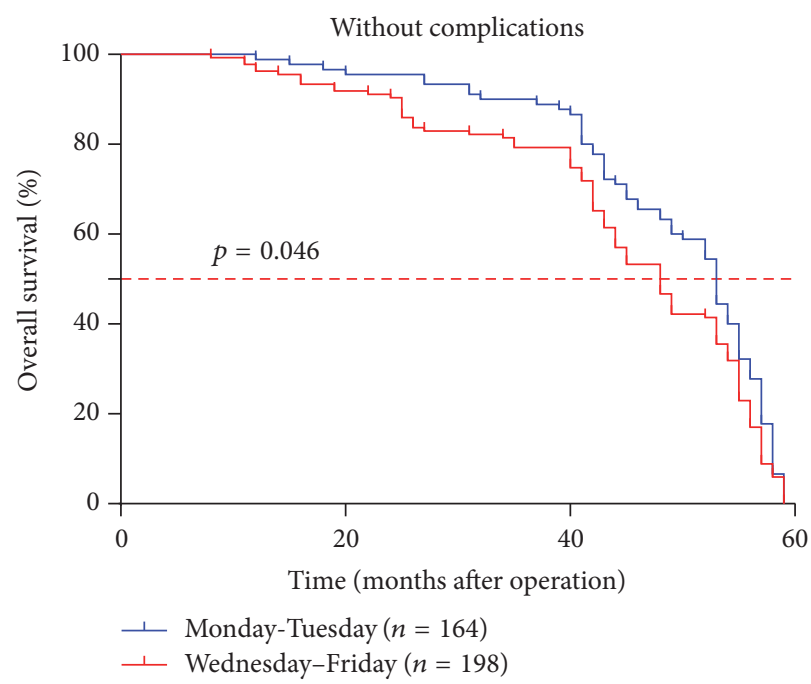

(c)

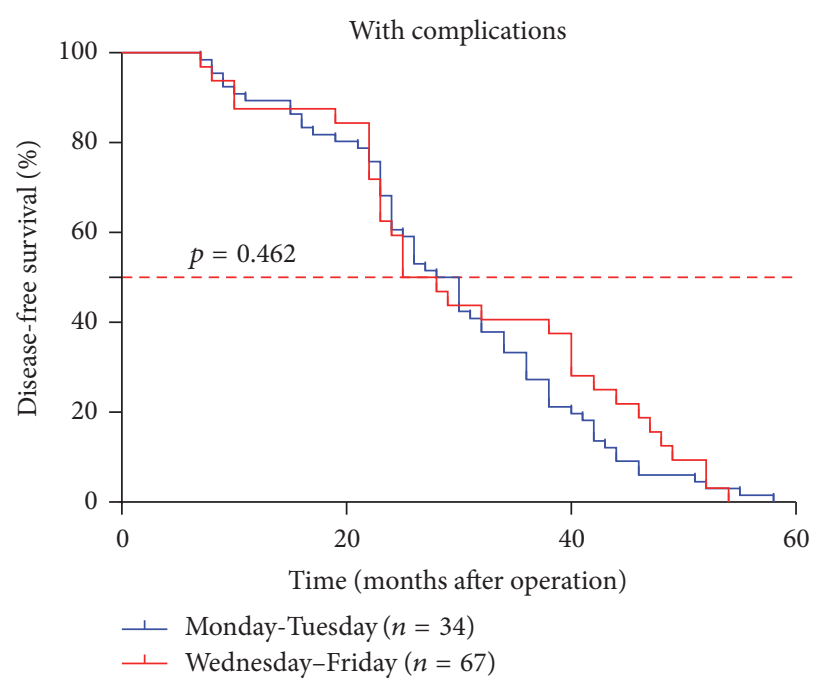

(b)

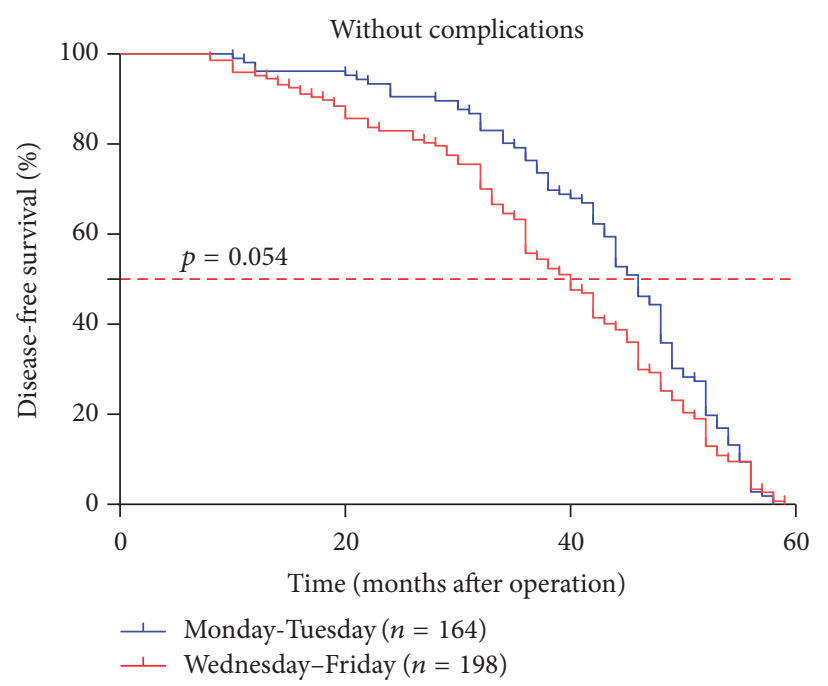

(d)

FIGURE 2: In the patients with postoperative complications, there was no significant difference between surgery on the earlier weekday and the later weekday in overall survival time and disease-free time $(\mathrm{a}, \mathrm{b})$. However, the weekday of surgery is still likely to influence the 5-year overall survival rate (c) in the patients without complications but not relapse rate (d).

adverse outcome of gastric surgery might be partly influenced by the alertness of the surgeon; thirdly, it was not difficult to deduce that a surgeon would find it much easier to concentrate on exhausting and demanding surgery in the earlier weekday. Above all, this hypothesis gained support from the findings that operative time and operative blood loss were increased in the later weekday slightly but significantly. Inadequate staffing on the following weekend after surgery is indeed one of the factors that should be considered, as complications were more likely picked up later with delayed medical care [27]. Moreover, the degree of complication severity was increased from Wednesday to Friday, compared to Monday. Even so, the effect of weekday of surgery or postoperative complications was weak in the patients with advanced tumor stage for the little chance of cure.
Moreover, from the present analysis, we found that preoperative comorbidity was a significant impact factor for morbidity, as described previously [28]. Beyond that, we also found that various influential factors are reported to increase the occurrence of complications after surgical resection, including old age, abnormal BMI, large tumor size, low serum albumin, longer duration of operation, and advanced tumor stage which was consistent with what has been reported before $[9,29]$. Accordingly, our results also showed that smoking had an obvious influence on the occurrence of complications; we could infer that except the sensitive respiratory system, nicotine addiction can easily induce an inflammatory state, thus impairing the body repairing and healing ability $[30,31]$. In contrast to the proposed "obesity paradox" [6] that overweight and obesity promoted better outcomes in patients 


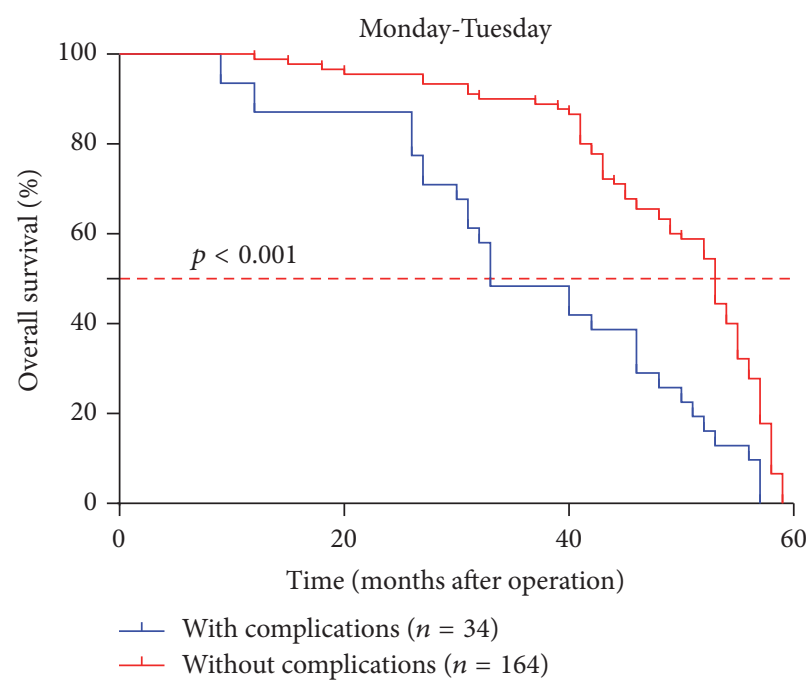

(a)

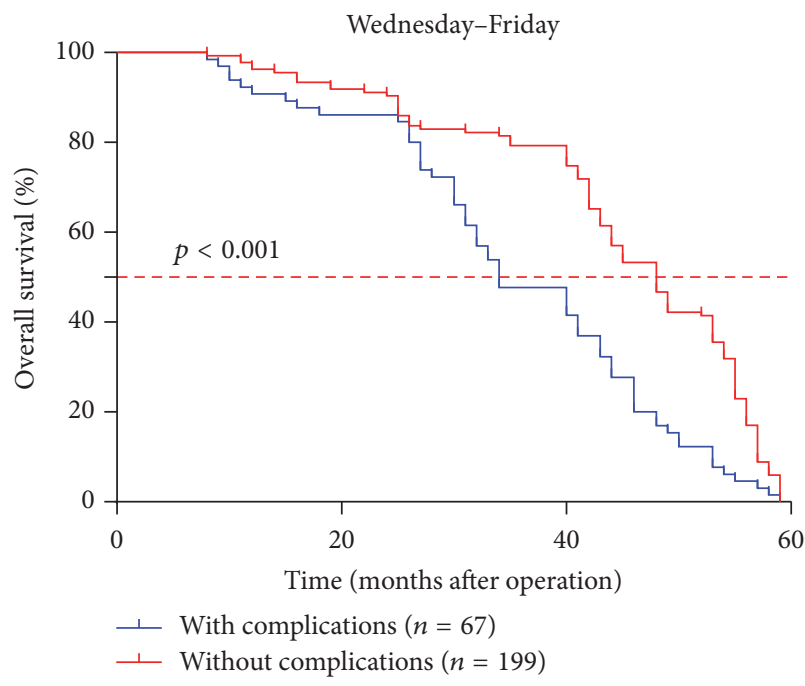

(c)

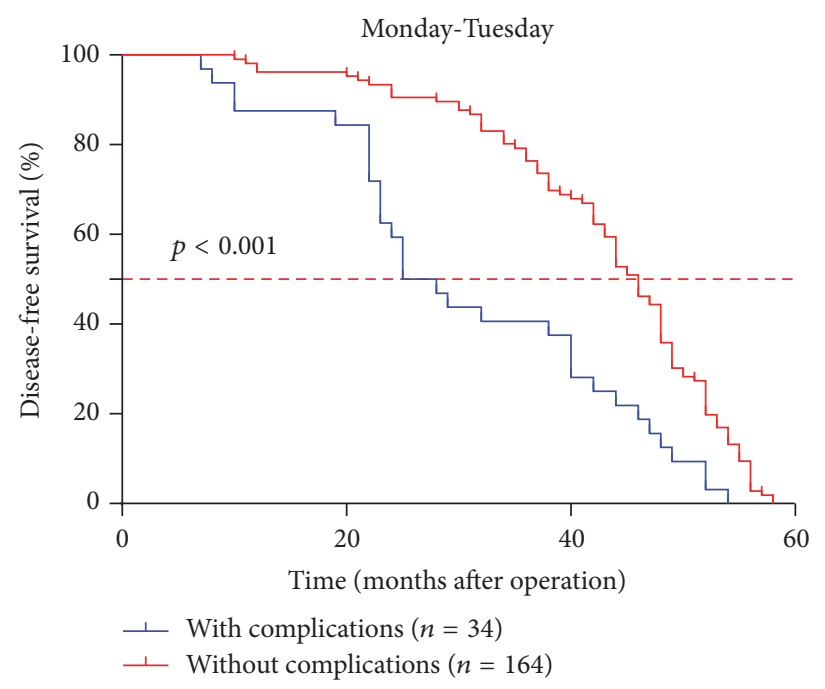

(b)

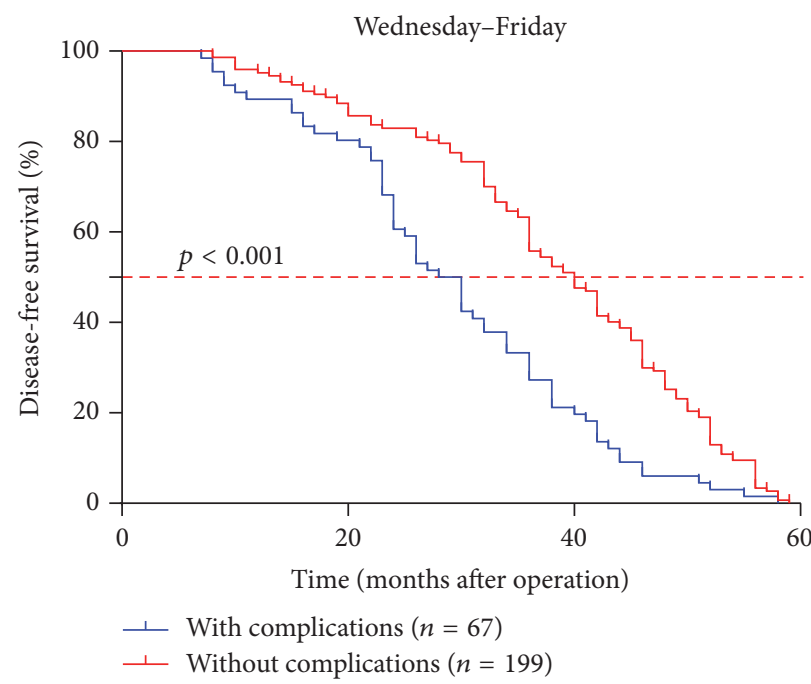

(d)

FIGURE 3: Regardless of weekday of surgery, the postoperative complications have a significant influence on the overall survival time and disease-free time after gastrectomy.

after gastrectomy, our study revealed that patients with high BMI had an higher morbidity after the gastric surgery; the increased rate was mainly due to the fact that excess fat may increase operation difficulty, operative time, and wound fat liquefaction as well, whose effects were significant in our study. Moreover, advanced tumor stage accompanied with more intraoperative blood loss, complicated operative procedure, and intensive shock was demonstrated to facilitate postoperative complications.

In the present study, preoperative comorbidity was responsible for the medical complications, especially in those with diabetes mellitus, which increased the incidence of infectious complications by more than 5 times. Preoperative coexisted active hepatic or nephrotic disease was obligated for most Grade IV complications in our study. Moreover, our results revealed that it was the severity grade of postoperative complications that promoted distinct clinical outcomes, but not complications type. Furthermore, the old age also represented a synergetic role with the postoperative complications in deteriorating the outcome. These results offered crucial clinical implications for selecting appropriate patients for surgery to reduce the postoperative morbidity. In conclusion, our present study indicated that the surgery performed on the later weekday associated with an increased postoperative morbidity and predicted a poor prognosis after gastric cancer surgery. Gastric cancer surgery for more readily surgically curable tumor stages (I-II) was followed by a less postoperative morbidity, relapse, and a better prognosis if conducted in the earlier weekday and the patient was well prepared. Thus, scheduling of gastric cancer surgery properly and a strict perioperative management to decrease the occurrence of postoperative complications might help to improve the prognosis in patients operated on for gastric cancer. 


\section{Conflicts of Interest}

The authors have no conflicts of interest.

\section{Acknowledgments}

This work was supported by grants from the National Natural Scientific Foundation of China (no. 81570509).

\section{References}

[1] W. Chen, R. Zheng, P. D. Baade et al., "Cancer statistics in China, 2015," CA Cancer Journal for Clinicians, vol. 66, no. 2, pp. 115$132,2016$.

[2] M. Isaka, H. Kondo, T. Maniwa, S. Takahashi, and Y. Ohde, "Boundary between N1 and N2 lymph node descriptors in the subcarinal zone in lower lobe lung cancer: a brief report," Journal of Thoracic Oncology, vol. 11, no. 7, pp. 1176-1180, 2016.

[3] S. Gao, J. Li, X. Feng, S. Shi, and J. He, "Characteristics and surgical outcomes for primary malignant melanoma of the esophagus," Scientific Reports, vol. 6, Article ID 23804, 2016.

[4] Y.-Q. Shi, J. Yang, P. Du et al., "Effect of Body Mass Index on Overall Survival of Pancreatic Cancer," Medicine (United States), vol. 95, no. 14, Article ID e3305, 2016.

[5] S. Murphy, G. Probert, J. Anderson et al., "Malignant mesothelioma, hypoalbuminaemia and the effect ofcarboplatin/pemetrexed on survival," Clinical Oncology, vol. 25, no. 12, pp. 713-718, 2013.

[6] H.-N. Chen, X.-Z. Chen, W.-H. Zhang et al., "The Impact of body mass index on the surgical outcomes of patients with gastric cancer a 10-year, single-institution cohort study," Medicine (United States), vol. 94, no. 42, p. e1769, 2015.

[7] G. de Manzoni and G. E. Verlato, "Gastrectomy with extended lymphadenectomy for primary treatment of gastric cancer $\mathrm{Br} \mathrm{J}$ Surg (Br J Surg 2005; 92: 5-13)," British Journal of Surgery, vol. 92, no. 6, p. 784, 2005.

[8] J. Y. Cho, H.-S. Han, Y.-S. Yoon, D. W. Hwang, K. Jung, and Y. K. Kim, "Postoperative complications influence prognosis and recurrence patterns in periampullary cancer," World Journal of Surgery, vol. 37, no. 9, pp. 2234-2241, 2013.

[9] X. Xia, W. Wu, K. Zhang et al., "Prognostic significance of complications after laparoscopic colectomy for colon cancer," PLoS ONE, vol. 9, no. 10, Article ID e108348, 2014.

[10] J. Szkandera, A. Gerger, B. Liegl-Atzwanger et al., "Pretreatment anemia is a poor prognostic factor in soft tissue sarcoma patients," PLoS ONE, vol. 9, no. 9, Article ID e107297, 2014.

[11] P. Aylin, R. Alexandrescu, M. H. Jen, and et al., "Day of week of procedure and 30 day mortality for elective surgery: retrospective analysis of hospital episode statistics," British Medical Journal, vol. 346, Article ID f2424, 2013.

[12] J. Lagergren, F. Mattsson, and P. Lagergren, "Weekday of esophageal cancer surgery and its relation to prognosis," Annals of Surgery, vol. 263, no. 6, pp. 1133-1137, 2016.

[13] M. M. Zare, K. M. F. Itani, T. L. Schifftner, W. G. Henderson, and S. F. Khuri, "Mortality after nonemergent major surgery performed on friday versus monday through wednesday," Annals of Surgery, vol. 246, no. 5, pp. 866-874, 2007.

[14] H.-N. Chen, X.-Z. Chen, W.-H. Zhang et al., "Necessity of harvesting at least 25 lymph nodes in patients with stage N2N3 resectable gastric cancer: a 10-year, single-institution cohort study," Medicine (Baltimore), vol. 94, no. 10, article e620, 2015.
[15] K. Washington, "7th edition of the AJCC cancer staging manual: stomach," Annals of Surgical Oncology, vol. 17, no. 12, pp. 30773079, 2010.

[16] D. Dindo, N. Demartines, and P.-A. Clavien, "Classification of surgical complications: a new proposal with evaluation in a cohort of 6336 patients and results of a survey," Annals of Surgery, vol. 240, no. 2, pp. 205-213, 2004.

[17] B. A. Chan, R. W. Jang, R. K. Wong, and et al., "Improving outcomes in resectable gastric cancer: a review of current and future strategies," Oncology (Williston Park), vol. 30, pp. 635645, 2016.

[18] T. Nishida and T. Doi, "Improving prognosis after surgery for gastric cancer," The Lancet Oncology, vol. 15, no. 12, pp. 12901291, 2014.

[19] S. P. Sussenbach, E. N. Silva, M. A. Pufal, D. S. Casagrande, A. V. Padoin, and C. C. Mottin, "Systematic review of economic evaluation of laparotomy versus laparoscopy for patients submitted to Roux-en-Y gastric bypass," PLoS ONE, vol. 9, no. 6, Article ID e99976, 2014.

[20] A. Andalib, A. V. Ramana-Kumar, G. Bartlett, E. L. Franco, and L. E. Ferri, "Influence of postoperative infectious complications on long-term survival of lung cancer patients: A populationbased cohort study," Journal of Thoracic Oncology, vol. 8, no. 5, pp. 554-561, 2013.

[21] B. K. Sah, Z. G. Zhu, M. M. Chen et al., "Effect of surgical work volume on postoperative complication: superiority of specialized center in gastric cancer treatment," Langenbeck's Archives of Surgery, vol. 394, no. 1, pp. 41-47, 2009.

[22] S. C. Katz, J. Shia, K. H. Liau et al., "Operative blood loss independently predicts recurrence and survival after resection of hepatocellular carcinoma," Annals of Surgery, vol. 249, no. 4, pp. 617-623, 2009.

[23] N. Blumberg, J. Heal, C. Chuang, P. Murphy, and M. Agarwal, "Further evidence supporting a cause and effect relationship between blood transfusion and earlier cancer recurrence," Annals of Surgery, vol. 207, no. 4, pp. 410-415, 1988.

[24] F. Balkwill and A. Mantovani, "Inflammation and cancer: back to Virchow?” Lancet, vol. 357, no. 9255, pp. 539-545, 2001.

[25] C. Menetrier-Caux, G. Montmain, M. C. Dieu et al., "Inhibition of the differentiation of dendritic cells from CD34(+) progenitors by tumor cells: role of interleukin- 6 and macrophage colony-stimulating factor," Blood, vol. 92, no. 12, pp. 4778-4791, 1998.

[26] F. Horn, C. Henze, and K. Heidrich, "Interleukin-6 signal transduction and lymphocyte function," Immunobiology, vol. 202, no. 2, pp. 151-167, 2000.

[27] D. I. McIsaac, G. L. Bryson, and C. van Walraven, "Elective, major noncardiac surgery on the weekend: a population-based cohort study of 30-day mortality," Medical Care, vol. 52, pp. 557$564,2014$.

[28] N. Brusselaers, F. Mattsson, M. Lindblad, and J. Lagergren, "Association between education level and prognosis after esophageal cancer surgery: a Swedish population-based cohort study," PLoS ONE, vol. 10, no. 3, Article ID e0121928, 2015.

[29] D. Marrelli, C. Pedrazzani, A. Neri et al., "Complications after extended (D2) and superextended (D3) lymphadenectomy for gastric cancer: analysis of potential risk factors," Annals of Surgical Oncology, vol. 14, no. 1, pp. 25-33, 2007.

[30] K. H. Jung, S. M. Kim, M. G. Choi et al., "Preoperative smoking cessation can reduce postoperative complications in gastric cancer surgery," Gastric Cancer, vol. 18, no. 4, pp. 683-690, 2014. 
[31] Y. Zheng, X. Cao, J. Wen et al., "Smoking affects treatment outcome in patients with resected esophageal squamous cell carcinoma who received chemotherapy," PLoS ONE, vol. 10, no. 4, Article ID e0123246, 2015. 


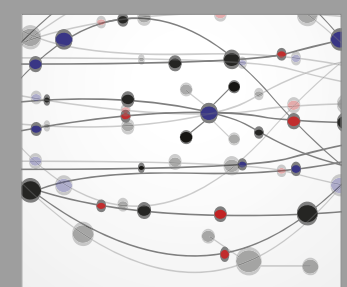

The Scientific World Journal
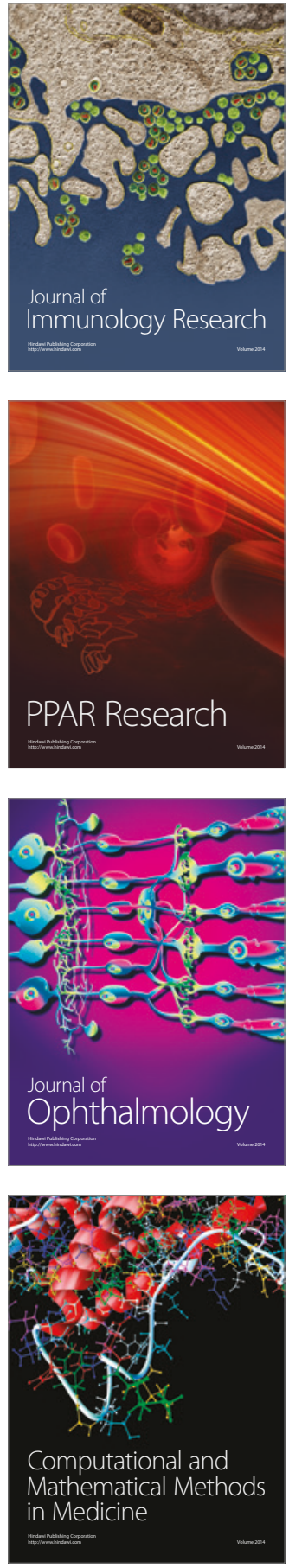

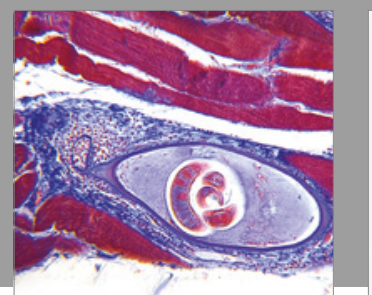

Gastroenterology Research and Practice
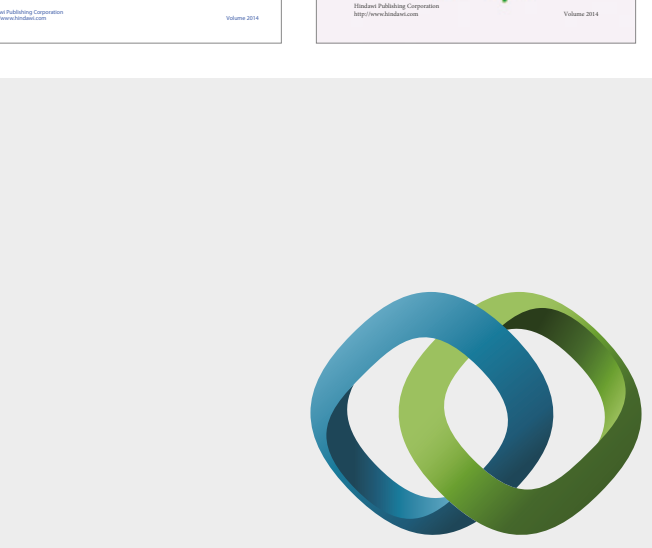

\section{Hindawi}

Submit your manuscripts at

https://www.hindawi.com
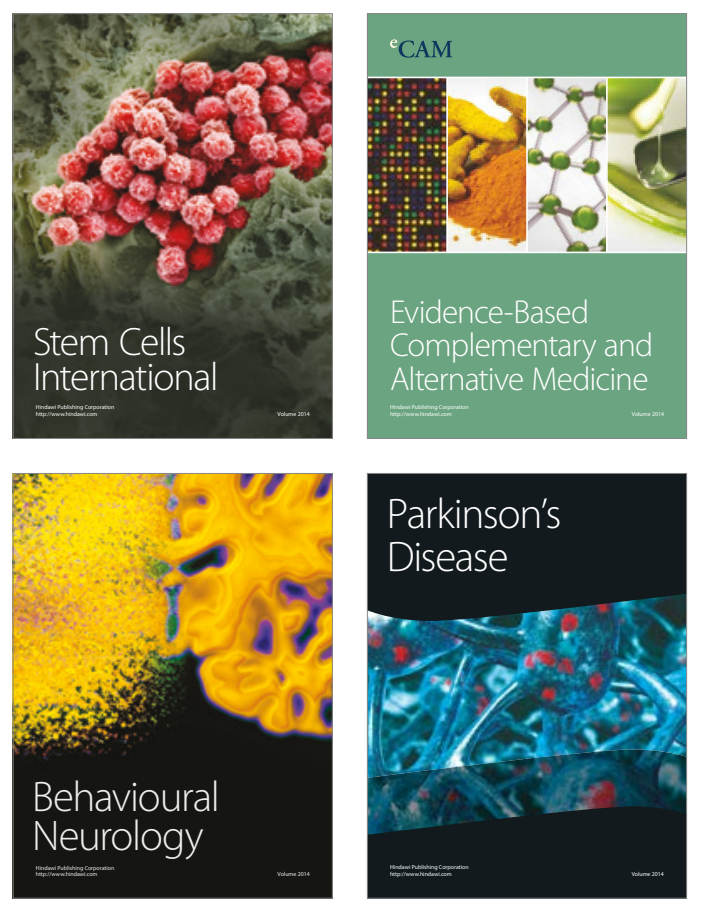
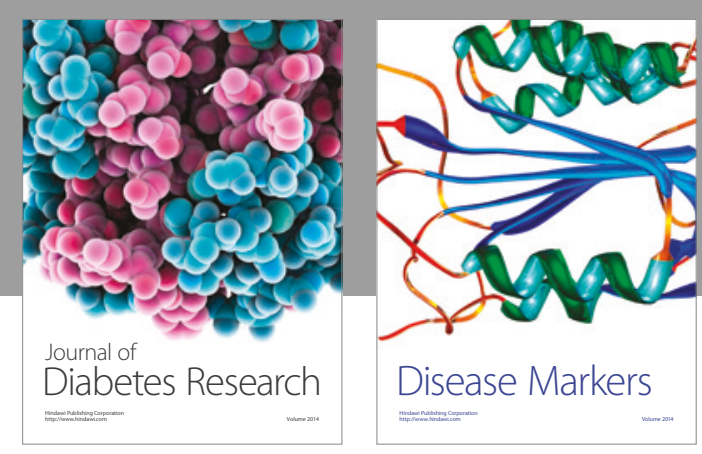

Disease Markers
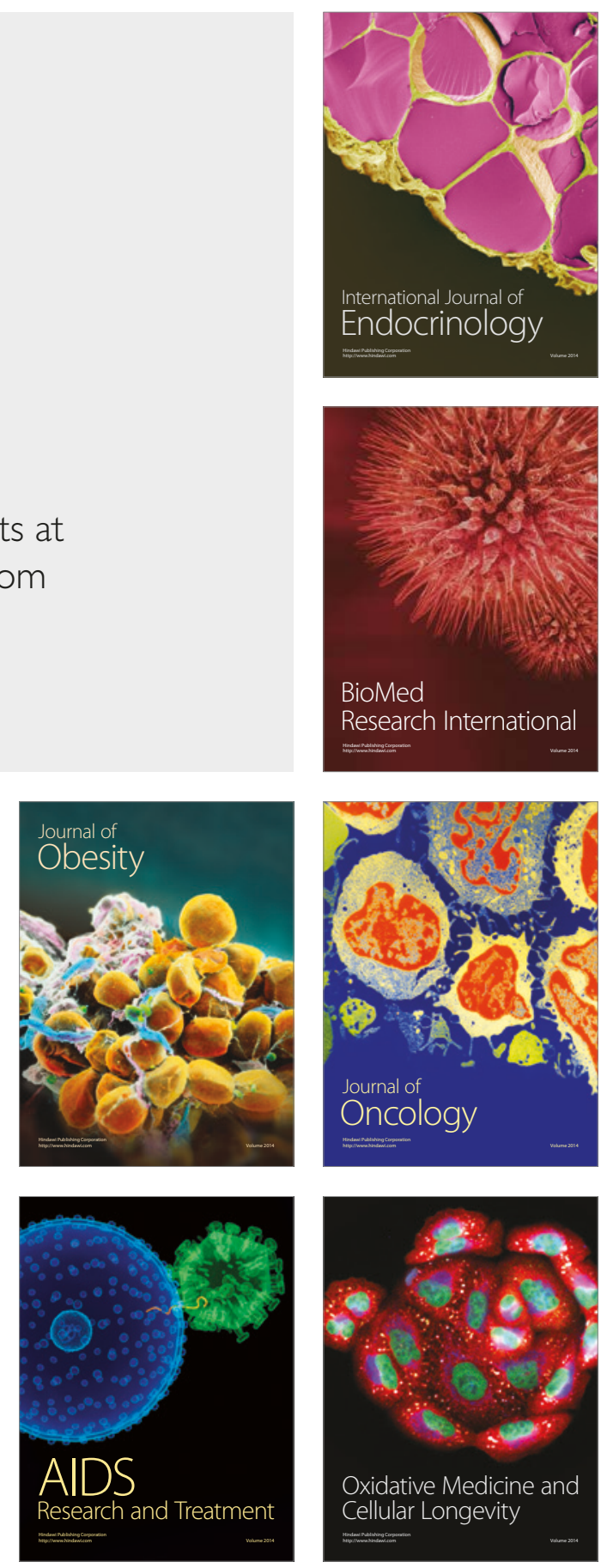